\title{
High performance of electrochemical sensors based on LbL films of gold nanoparticles, polyaniline and sodium montmorillonite clay mineral for simultaneous detection of metal ions
}

\author{
Anerise de Barros ${ }^{\mathrm{a}, \mathrm{b}}$, Carlos José Leopoldo Constantino ${ }^{\mathrm{c}}$, Nilson Cristino da Cruz $^{\mathrm{d}}$, \\ José Roberto Ribeiro Bortoleto ${ }^{\mathrm{d}}$, Marystela Ferreira ${ }^{\mathrm{b}, \mathrm{c}, *}$ \\ ${ }^{a}$ Faculdade de Ciências, UNESP/POSMAT, Univ Estadual Paulista, Bauru, SP, Brazil \\ ${ }^{\mathrm{b}}$ Universidade Federal de São Carlos, UFSCar, Campus Sorocaba, SP, Brazil \\ ${ }^{\mathrm{c}}$ Faculdade de Ciênciase Tecnologia, UNESP Univ Estadual Paulista, Presidente Prudente, SP, Brazil \\ d Campus Experimental de Sorocaba, UNESP Univ Estadual Paulista, Sorocaba, SP, Brazil
}

\section{A R T I C L E I N F O}

\section{Article history:}

Received 6 February 2017

Received in revised form 17 March 2017

Accepted 18 March 2017

Available online 20 March 2017

\section{Keywords:}

Layer-by-Layer film

gold nanoparticles

polyaniline

sodium montmorilonitte clay

electrochemical sensors

metal ion detection

\begin{abstract}
A B S T R A C T
We report the effects of the incorporation of gold nanoparticles (AuNPs) into LbL films of emeraldine salt polyaniline (PAni-ES) and sodium montmorillonite clay mineral $\left(\mathrm{Na}^{+}-\mathrm{MMT}\right)$. We observed the higher performance of electrochemical sensors was induced by AuNPs towards trace level detection of cadmium $\left(\mathrm{Cd}^{2+}\right)$, lead $\left(\mathrm{Pb}^{2+}\right)$ and copper $\left(\mathrm{Cu}^{2+}\right)$ ions. The detection was performed by square wave anodic stripping voltammetry (SWASV) using ITO electrodes modified with LbL films composed of three supramolecular architectures: PAni-ES/AuNPs, AuNPs/ $/ \mathrm{Na}^{+}$-MMT and PAni-ES/AuNPs $/ \mathrm{Na}^{+}-\mathrm{MMT}$, which were compared to the LbL films without AuNPs. The results are consistent with the AFM dates indicating that the increase of roughness favors at low limit of detection for the sensors. The incorporation of AuNPs and PAni-ES leads to an interaction via ion-dipole, as characterized by FTIR and Raman spectra. This interaction, consequently, causes a more extended polymer chain, which intercalate into $\mathrm{Na}^{+}$-MMT galleries, according to X-ray diffraction data. The results demonstrate the possible control of film properties by exploiting molecular-level interactions in multicomponent nanostructured films favoring directly in electrochemical sensors response.
\end{abstract}

(C) 2017 Elsevier Ltd. All rights reserved.

\section{Introduction}

Enormous efforts have been devoted to developing new materials where molecular control is key for enhanced sensing performance. In spite of the variety of contributions in this field, monitoring of the environment still poses challenges that are worth pursuing. In many cases, sensing is made with films containing mercury or using mercury electrodes, which are not environmentally friendly and may be harmful to human health. Carbon paste electrodes have been used, with little or no impact to the environment, but then the amount of material used is much larger than in nanostructured films. In this context, the Layer-by-Layer ( $\mathrm{LbL}$ ) technique is very interesting for producing

\footnotetext{
* Corresponding author at: Universidade Federal de São Carlos, UFSCar, Campus Sorocaba, SP, Brazil. Tel.: +55 153229 8810, Fax: +55 1532296000 .

E-mail address: marystela@ufscar.br (M. Ferreira).
}

supramolecular structure due to its ability to combine different materials in a synergistic way [1-4]. Various examples may be given of controlled molecular architectures built with LbL films, such as optical, electrical and magnetic properties, could be tailored [3-7]. With the efficient control that highlighted these properties, it is possible to obtain a wide range of applications with these LbL films, especially for sensing and biosensing [5,812]. For example, the incorporation of gold nanoparticles (AuNRs) in sensors and biosensors have been highly attractive due to their chemical stability, biocompatibility and high catalytic activity for various chemical and electrochemical process [5,13-17]. For instance, Berzina et al. [18] evaluated the electrical properties of PAni-ES induced by AuNPs caped with mercapthoethanesulfonic. The interactions between materials were obtained by in situ polymerization and stability via sulfonic group that caped the AuNPs. The authors verify, by means of nonlinear electrical measurements, that the direct 


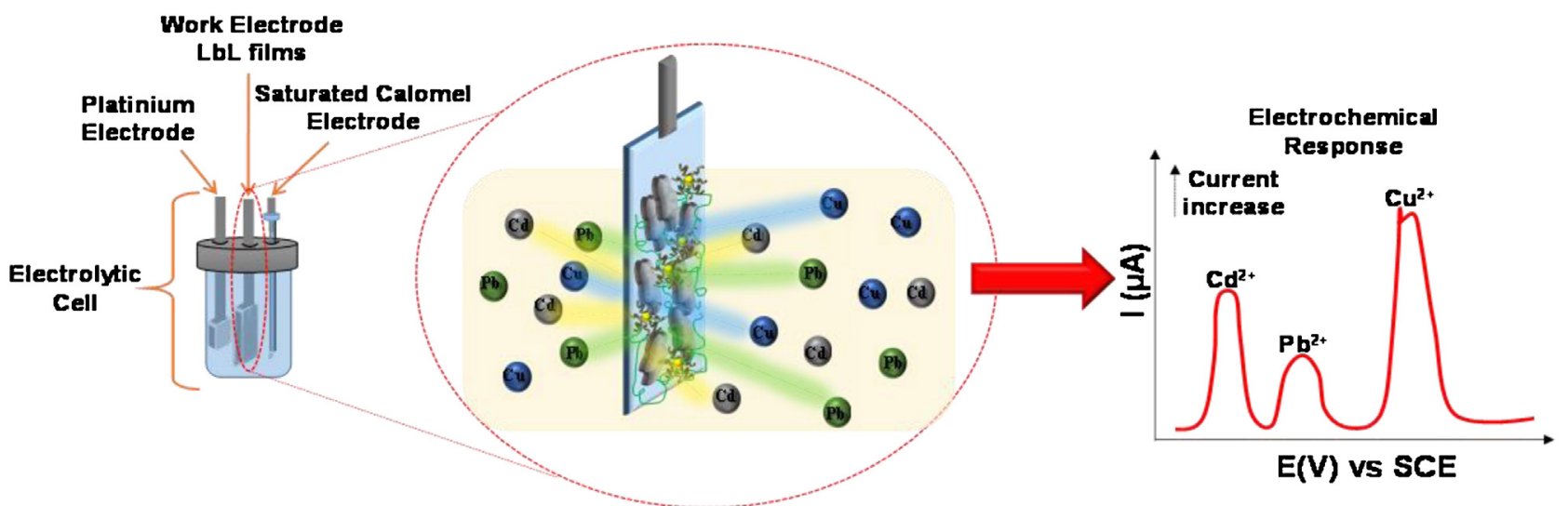

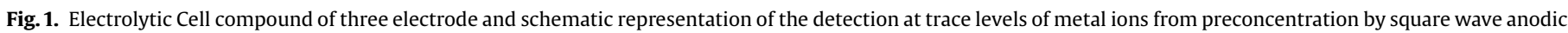
stripping voltammetry.

addition of AuNPs into polymeric chain increases the conductive power of the polymer.

Gold nanoparticles, are also used in combination with organic materials such as graphene and carbon nanotubes, increase the catalytic activity of the sensors [5,15]. For example, Savafi and Farjami [19] fabricated a carbon electrode with AuNPs thiolated for detecting Mercury traces. The authors verify the higher performance of the sensors with the incorporation of
AuNPs when compared to neat carbon electrode, showing the limit of detection of $2.3 \mathrm{nmol} \mathrm{L}^{-1}$. Electrochemical sensors composed of graphene supported in AuNPs were fabricates through the LbL technique for detecting $\mathrm{H}_{2} \mathrm{O}_{2}$. Electrochemical results obtained by cyclic and amperometric voltammetry indicated the excellent performance of the electrodes with incorporated AuNPs and that they are very promising for detection of $\mathrm{H}_{2} \mathrm{O}_{2}$ [5].
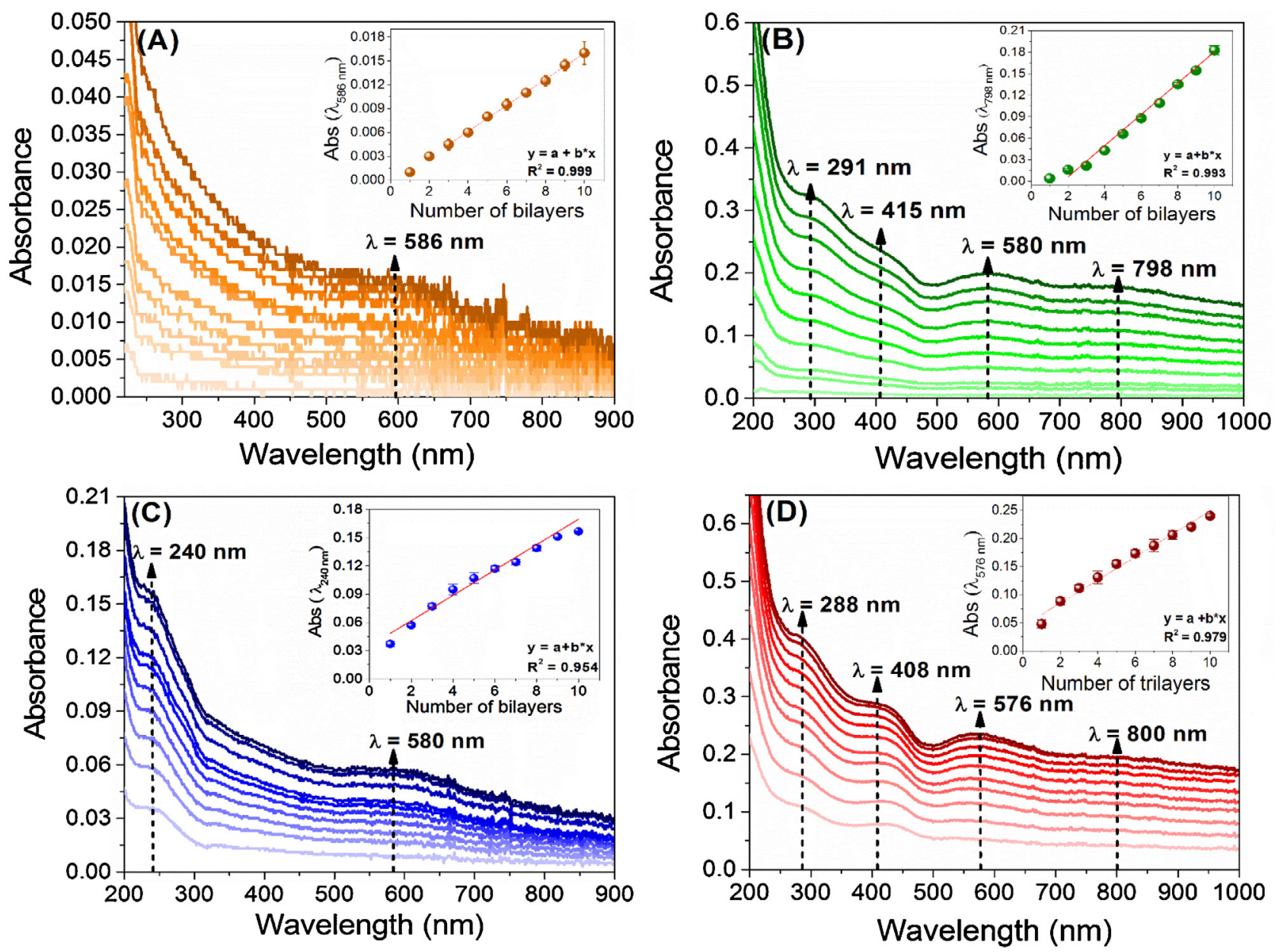

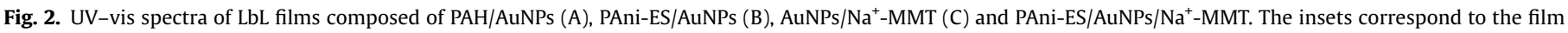
growth as a function of the number of bilayers for each architecture. 


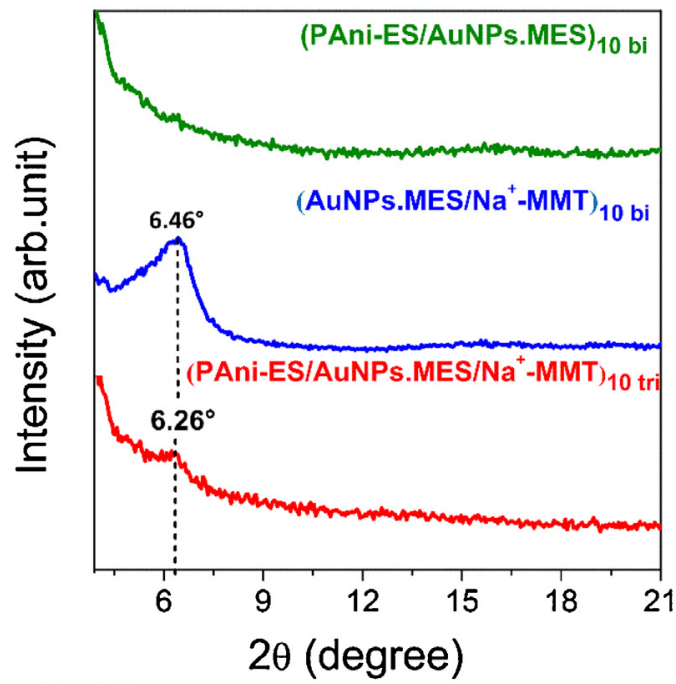

Fig. 3. XRD patterns of 10-bilayers LbL films of PAni-ES/AuNPs, AuNPs/Na ${ }^{+}-\mathrm{MMT}$ and 10-trilayers of PAni-ES/AuNPs/Na ${ }^{+}$-MMT deposited on silicon substrates.

Rao et al. [20]. modified glass carbon electrode with AuNPs and PAni-ES composites to increase the electrode sensitivity and to amplify the sensor signal for detection of melanine. The electrochemistry analysis platforms presents a low limit of detection of $1.39 \mathrm{pmol} \mathrm{L}^{-1}$. Electrochemical sensor composed o PAni electro-synthesized on the surface of screen-printed carbon electrodes in the presence of sodium dodecyl sulfate (SDS) as dopant. The PAni-SDS electrode modified was integrated into PDMS microfluidic chip to detection of mercury trace level with limit of detection of $2.4 \mathrm{nmol} \mathrm{L}^{-1}$ [21].

We aim here the development of composite materials where molecular control and nanostructuring obtained by the LbL technique to promote enhanced sensing performance. Furthermore, clays promote cationic change with metal ions while interaction of AuNPs and PAni-ES increase electrical conductivity, thus enhancing electron transfer and electrocatalytic effect. In earlier work we reported on effects of AuNPs in the LangmuirBlodgett (LB) films of PAni-ES and OMt clay that promoted stabilization of mixed composite Langmuir films and enhanced the electrocatalytic effect for detecting metal ions, yielding a higher performance with lower limit of detection [22]. Because this type of influence of AuNPs, here we studied the nanostructuring these materials from the LbL technique, where independently of different ordering we observed through LB and LbL techniques it is possible obtain the molecular interaction lows limit of detection is achieved. Most importantly, the incorporation of AuNPs indeed improved the performance of sensing devices in detecting trace levels of cadmium $\left(\mathrm{Cd}^{2+}\right)$, lead $\left(\mathrm{Pb}^{2+}\right)$ and copper $\left(\mathrm{Cu}^{2+}\right)$ ions, when

Table 1

Comparison of basal spacing values calculated for LbL films with and without AuNPs.

\begin{tabular}{|c|c|c|c|c|}
\hline \multirow{2}{*}{$\frac{\text { LbL Films }}{{ }^{\mathrm{a}} \text { PAni-ES/PVS }}$} & \multicolumn{3}{|c|}{$2 \theta$ (degree) } & \multirow{2}{*}{$\begin{array}{l}\text { Lamelar space }(\mathrm{nm}) \\
-\end{array}$} \\
\hline & 13.2 & - & - & \\
\hline PAni-ES/AuNPs & - & - & - & - \\
\hline${ }^{\mathrm{a}} \mathrm{PEI} / \mathrm{Na}^{+}-\mathrm{MMT}$ & 6.8 & 14.3 & 17.1 & 1.32 \\
\hline $\mathrm{AuNPs} / \mathrm{Na}^{+}-\mathrm{MMT}$ & 6.4 & - & - & 1.37 \\
\hline${ }^{\mathrm{a}} \mathrm{PAni}-\mathrm{ES} / \mathrm{Na}^{+}-\mathrm{MMT}$ & 7.3 & 14.5 & 17.4 & 1.21 \\
\hline PAni-ES/AuNPs/Na ${ }^{+}-M M T$ & 6.2 & - & - & 1.41 \\
\hline
\end{tabular}

\footnotetext{
a Values extracted from De Barros et al. [23].
}

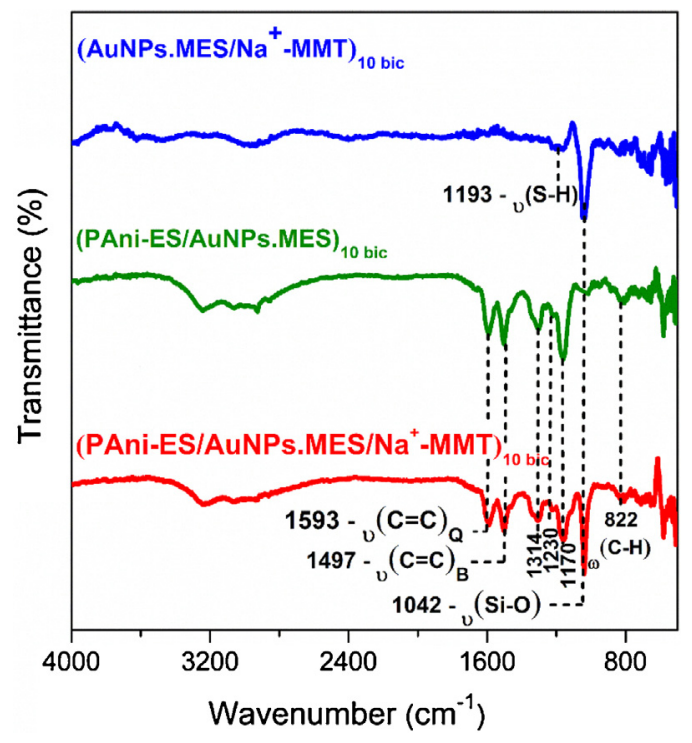

Fig. 4. FTIR spectra recorded in transmittance mode of 10-bilayers LbL films of PAni-ES/AuNPs, AuNPs/Na ${ }^{+}-\mathrm{MMT}$ and 10 -trilayers of PAni-ES/AuNPs/Na ${ }^{+}-\mathrm{MMT}$ deposited on silicon substrates.

compared with the sensing devices of PAni-ES and $\mathrm{Na}^{+}-\mathrm{MMT}$ without AuNPs [23].

\section{Materials and Methods}

\subsection{Materials}

Sodium montmorillonite clay mineral $\left(\mathrm{Na}^{+}-\mathrm{MMT}\right)$ was pur-

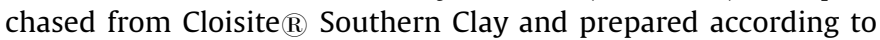
the method described by Umemura et al. [24]. The $\mathrm{Na}^{+}-\mathrm{MMT}$ $\left(\mathrm{Mw}=2892.48 \mathrm{~g} \cdot \mathrm{mol}^{-1}\right)$ were dispersed in ultrapure water with $\mathrm{pH}$ adjusted to 2.5 at $1.0 \mathrm{~g} \mathrm{~L}^{-1}$ and sonicated for 2 hours. PAni-ES $\left(\mathrm{MW}=362.0 \mathrm{~g} \mathrm{~mol}^{-1}\right.$, for each repeat unit) solution were prepared according to the methods described by Cheung et al. [25], which involved $0.5 \mathrm{~g}$ of emeraldine base (EB) diluted in $25.0 \mathrm{~mL}$ of $\mathrm{N}$ dimethylacetamide (DMAc). The solution was stirred overnight and further filtered. The doping process was made by adding $3.0 \mathrm{~mL}$ dropwise in $26.0 \mathrm{~mL}$ of $\mathrm{HCl}$ solution with $\mathrm{pH} 2.5$ under stirred during 10 minutes. Gold nanoparticles (AuNPs) were obtained based on the method described by Gofberg and Mandler [26], which involved $0.0394 \mathrm{~g}$ of $\mathrm{HAuCl}_{4}$ and $0.0164 \mathrm{~g}$ dissolved in $36.0 \mathrm{~mL}$ of water/acetic acid (final pH 3.0) and stirred for $10 \mathrm{~min}$ at room temperature. The nanoparticles were reduce by adding $7.6 \mathrm{mg} \mathrm{NaBH} 4$ dissolved in $10.0 \mathrm{~mL}$ of deionized water; the solution slowly turned into deep purple and was stirred for 2 hours. The AuNPs were precipitate by adding acetonitrile (ACN), after precipitation and decantation of ACN the sample was rinsed twice

Table 2

Assignment of the FTIR bands of the LbL films containing PEI/Na ${ }^{+}-\mathrm{MMT}$ compared with LbL film of AuNPs/Na ${ }^{+}-\mathrm{MMT}$.

\begin{tabular}{lll}
\hline \multirow{2}{*}{ Assignment } & Wavenumber $\left(\mathrm{cm}^{-1}\right)$ & \\
\cline { 2 - 3 } & ${ }^{\mathrm{a} P E I / \mathrm{Na}^{+}-\mathrm{MMT}}$ & AuNPs/Na ${ }^{+}-\mathrm{MMT}$ \\
\hline Stretching of OH group & 3627 & - \\
Stretching of Si-O group & 1040 & 1042 \\
Deformation of Al-O group & 526 & - \\
Deformation of Si-O group & 462 & - \\
\hline
\end{tabular}

a Values extracted from the reference De Barros et al. [23]. 
Table 3

Assignment of the FTIR bands for the LbL films containing PAni-ES/PVS and PAni-ES/Na ${ }^{+}-\mathrm{MMT}$ compared with LbL films PAni-ES/AuNPs and PAni-ES/AuNPs/Na ${ }^{+}$-MMT.

\begin{tabular}{|c|c|c|}
\hline \multirow[t]{2}{*}{ Assignment } & \multicolumn{2}{|l|}{ Wavenumber $\left(\mathrm{cm}^{-1}\right)$} \\
\hline & $\begin{array}{l}\text { PAni-ES/PVS } \\
{ }^{\text {a PAni-ES/Na }}{ }^{+}-\mathrm{MMT}\end{array}$ & $\begin{array}{l}\text { PAni-ES/AuNPs } \\
\text { PAni-ES/AuNPs/Na }{ }^{+}-\mathrm{MMT}\end{array}$ \\
\hline Stretching of $\mathrm{C}-\mathrm{C}$ benzenoid & 1493 & 1497 \\
\hline Stretching of secondary amine & 1325 & 1314 \\
\hline Stretching of radical cation $C-N^{+}\left(Q=N^{+} H-B\right.$ or $\left.B-N^{+} H-B\right)$ & 1246 & 1042 \\
\hline Vibration of $N=Q=N$ ip & 1159 & 1230 \\
\hline Deformation $\mathrm{C}-\mathrm{H}$ (ring benzenoid) op & 815 & 822 \\
\hline
\end{tabular}

a Corresponds to the reference in which the values were extracted are previously described.

with $5.0 \mathrm{~mL}$ of ethanol and decanted. The sample was subsequently suspended in aqueous solution at $\mathrm{pH} 2.5$; more information regarding the characterization of these nanoparticles can be seen in de Barros et al. [23,27] Poly(allylamine hydrochloride) (MW $\sim 17500 \mathrm{~g} \mathrm{~mol}^{-1}$ ) was purchased from Sigma-Aldrich and used as a polyelectrolyte support for the LbL films composed of AuNPs, with the $0.5 \mathrm{mg} \mathrm{mL}^{-1}$ concentration. Metal ions solutions $\left(\mathrm{CdCl}_{2} \cdot \mathrm{H}_{2} \mathrm{O}\right.$, $\mathrm{CuCl}_{2} \cdot \mathrm{H}_{2}$ ) and $\mathrm{PbCl}_{2}$ ) were purchased from Vetec and samples were prepared using a stock solution at $2000.0 \mu \mathrm{gL}^{-1}$ for the copper salt, $10.0 \mu \mathrm{g} \mathrm{L}^{-1}$ for the lead salt and $5.0 \mu \mathrm{g} . \mathrm{L}^{-1}$ for the cadmium salt.

\subsection{LbL films Fabrication}

The LbL films were fabricated in four types of architectures PAH

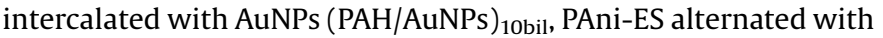
AuNPs (PAni-ES/AuNPs) 10bil, AuNPs intercalated with $\mathrm{Na}^{+}-\mathrm{MMT}$ and supported in one layer of PAH before adsorption of AuNPs (AuNPs/Na $\left.{ }^{+}-\mathrm{MMT}\right)_{10 b i l}$ and nanocomposite film composed by multilayers alternated with PAni-ES, AuNPs and $\mathrm{Na}^{+}-\mathrm{MMT}$ (PAniES/AuNPs/Na ${ }^{+}$-MMT) $)_{10 \text { tril. }}$ The different architectures were fabricate by dipping the substrates for 3 minutes in the PAni-ES solution, $5 \mathrm{~min}$ in AuNPs colloidal dispersion and $10 \mathrm{~min}$ in the $\mathrm{Na}^{+}$-MMT dispersion. Afterwards, the substrates were immersed for 30 seconds in washing solutions to remove the excess materials. With the four architectures, it was possible to verify whether there was synergy in the fabrication of nanocomposites and to conduct a comparative study. The LbL films were deposited in different substrates for UV-vis characterization using quartz plates, FTIR, Raman and XRD using silicon substrates, and for the electrochemical analysis, the films were deposited onto ITO (indium tin oxide) substrates.

\subsection{Characterization}

The UV-vis absorption spectra from 190 to $1000 \mathrm{~nm}$ of each deposited bilayer of the LbL films were obtain using a Genisys Thermo Scientific Spectrometer, model 6. Ten bilayers of $\mathrm{PAH} /$ AuNPs, PAni-ES/AuNPs and AuNPs/Na ${ }^{+}-\mathrm{MMT}$ and ten trilayers of PAni-ES/AuNPs/Na ${ }^{+}$-MMT were characterized by Fourier Infrared Spectroscopy (FTIR) using a Thermo Nicolet Spectrometer, model Nexus 470. The Raman spectra of the LbL films were obtained using a micro-Raman Renishaw spectrograph model in-Via, equipped with an 1800 grooves $/ \mathrm{mm}$ grating, CCD detector, laser at $633 \mathrm{~nm}$ and a Leica microscope which $50 \times$ objective lens collects spectra with ca. $1.0 \mu \mathrm{m}^{2}$ spatial resolution. The collecting time was $10 \mathrm{~s}$ and the spectral resolution was ca. $4.0 \mathrm{~cm}^{-1}$. X-ray diffraction experiments (XRD) were made using a PanAlytical diffractometer $(\mathrm{CuK} \alpha$, $\lambda=0.154 \mathrm{~nm}$ ) model ' $\mathrm{X}$ ' PertPro, for 10-layers LbL films deposited onto silicon substrates. The spectra were taken with a 0.05 step size, $3 \mathrm{~s}$ per step, voltage of $45 \mathrm{kV}$ and current of $30 \mathrm{~mA}$. The basal distances were calculated based on the peak position using the
Bragg equation. The surface topography of the samples was studied with an atomic force microscopy (AFM, XE-100, Park Systems) operating in the non-contact mode and using silicon cantilever whose nominal radius was $5.0 \mathrm{~nm}$. An area of $10.0 \mu \mathrm{m} \times 10.0 \mu \mathrm{m}$ was scanned at a resolution of $512 \times 512$ points. Based on the AFM images, the surface root mean squared (RMS) roughness was quantified. The voltammetry analysis was performed using a saturated calomel reference electrode (SCE) and a platinum counter electrode with an area of $1.0 \mathrm{~cm}^{2}$ with the LbL films
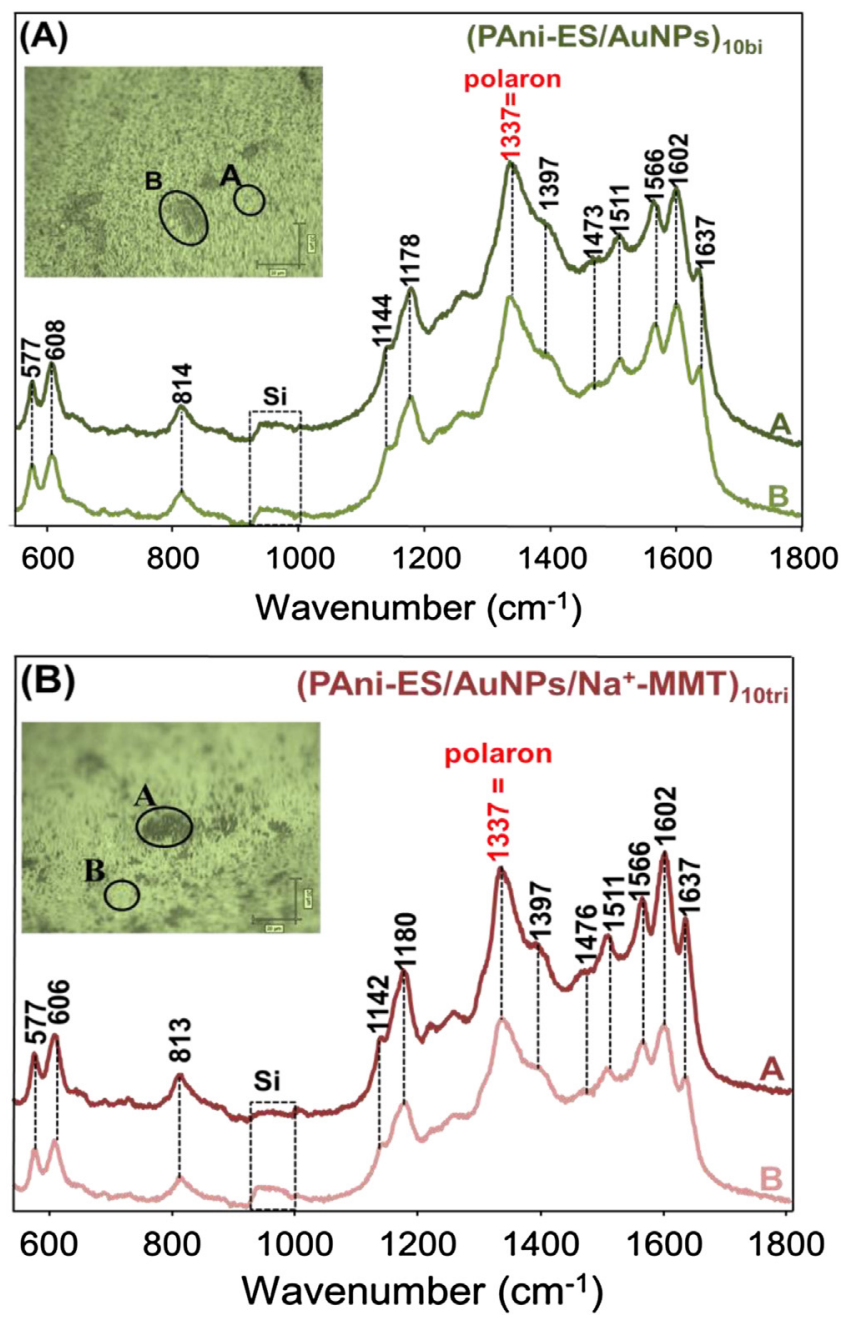

Fig. 5. Raman spectra of 10-bilayers LbL films of PAni-ES/AuNPs (A) and 10-trilayers LbL film of PAni-ES/AuNPs/Na+-MMT(B), deposited onto silicon substrates. The Raman spectra were recorded with 633 laser line. Insets: optical images of 10bilayers of PAni-ES/AuNPs and 10-trilayers of PAni-ES/AuNPs/Na ${ }^{+}-\mathrm{MMT}$ LbL films showing the spots where the Raman spectra were recorded. 

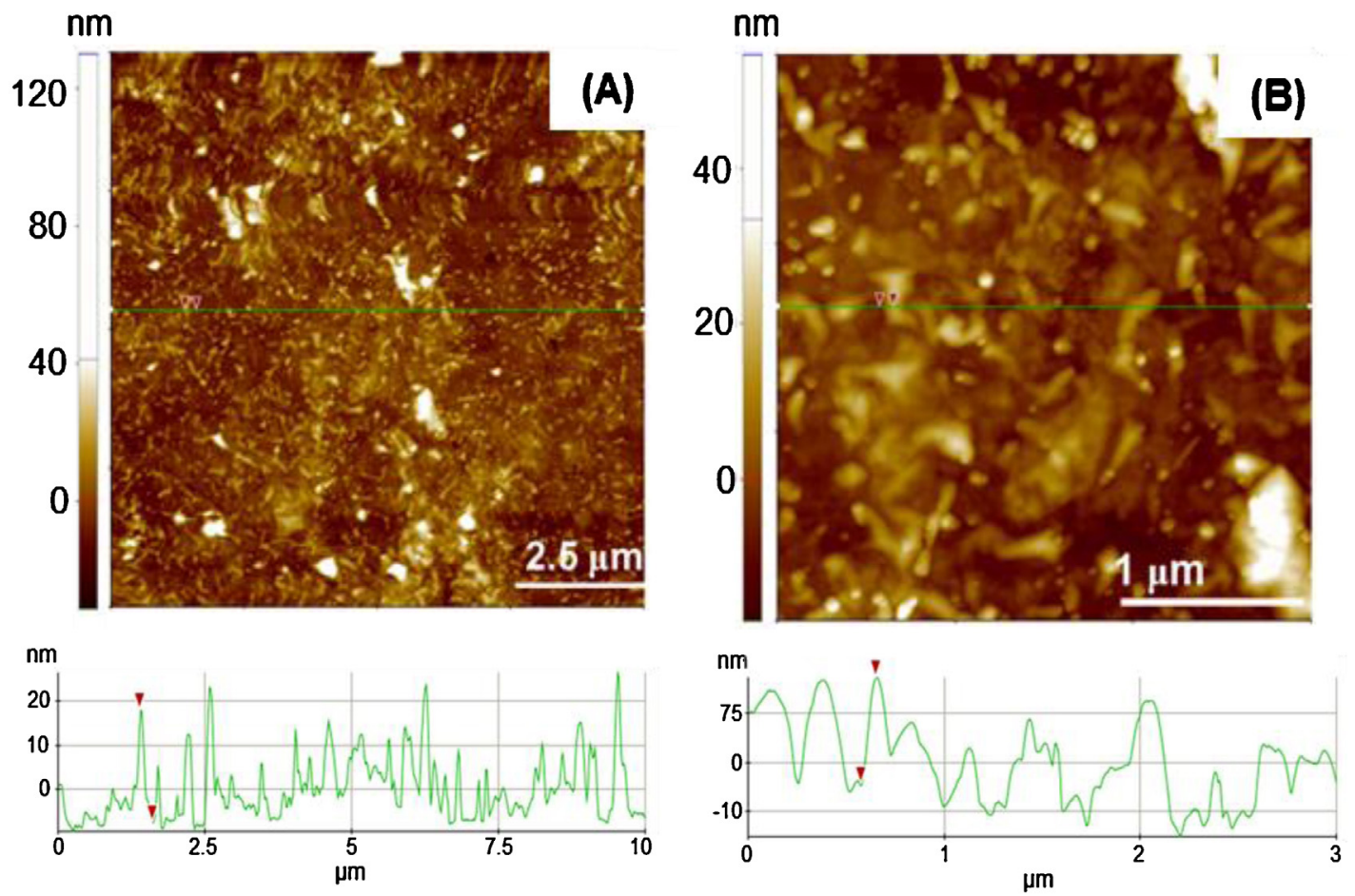

\begin{tabular}{|crrr|}
\hline Cursor & $\Delta X(\mu m)$ & $\Delta Y(n m)$ & \multicolumn{1}{c}{ Angle(deg) } \\
Red & 0.215 & -25.528 & -6.776 \\
\hline
\end{tabular}

\begin{tabular}{|rrrr|}
\hline Cursor & $\Delta X(\mu m)$ & \multicolumn{1}{c}{$\Delta Y(\mathrm{~nm})$} & \multicolumn{1}{c}{ Angle(deg) } \\
ERed & 0.076 & 21.698 & 15.900
\end{tabular}
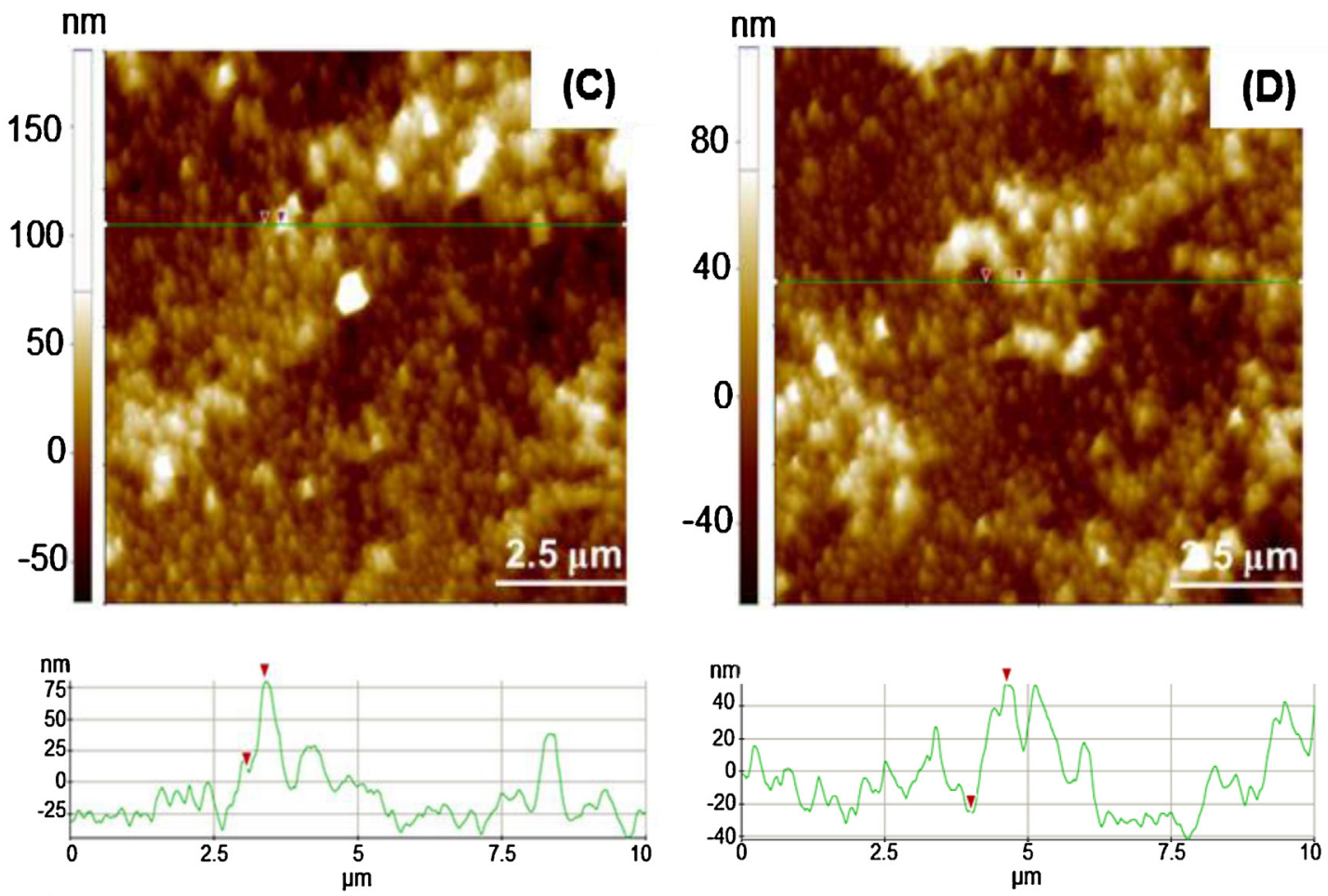

\begin{tabular}{|c|c|c|c|}
\hline Cursor & $\Delta x(u m)$ & $\Delta Y(\mathrm{~mm})$ & Ande(deg) \\
\hline Ined & 0.312 & 70.668 & 12.742 \\
\hline
\end{tabular}

\begin{tabular}{|rrrr|}
\hline Cursor & $\Delta X(\mu \mathrm{m})$ & \multicolumn{1}{c}{$\Delta Y(\mathrm{~nm})$} & \multicolumn{1}{l|}{ Angle(deg) } \\
Red & 0.625 & 78.015 & 7.115 \\
\hline
\end{tabular}

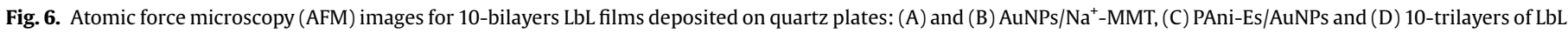
film PAni-ES/AuNPs/ $\mathrm{Na}^{+}-\mathrm{MMT}$. 
deposited on ITO substrate and covering $1.5 \mathrm{~cm}^{2}$ of the working electrode. For these analyses, it has been used a Potentiostat/ Galvanostat, model PGSTAT 30, coupled to an electrochemical cell, with measurements performed by square wave anodic stripping voltammetry (SWASV). The schematic mechanism of detection metal ions traces is depicted in Fig. 1.

\section{Results and Discussion}

\subsection{Characterization $\mathrm{LbL}$ films}

The UV-vis spectra in Fig. 2 show increasing absorbance with the number of bilayers or trilayers, with a linear growth for all LbL films (see the inset). The spectra for the PAH/AuNPs architecture in Fig. 2(A) exhibit a plasmon resonance band at $586 \mathrm{~nm}$ due to AuNPs, which is relatively weak as the AuNPs were capped by mercapethanethiol (MES) used for stabilization. AuNPs and $\mathrm{Na}^{+}-$ MMT have negative charge [26,28-30], but it was possible to growth the LbL films whose spectra in Fig. 2(C) display bands from both materials. In this case, we suggest that the adsorption between of $\mathrm{Na}^{+}-\mathrm{MMt}$ and AuNPs occurs via hydrogen interaction. Bands are observed at $240 \mathrm{~nm}$ and $580 \mathrm{~nm}$, which correspond to the charge transfer transition in the $\mathrm{Fe}-\mathrm{OH}$ group in $\mathrm{Na}^{+}-\mathrm{MMT}$ and the plasmonic band of AuNPs, respectively [23,27]. The LbL films containing PAni-ES had spectra dominated by the multiple bands of this polymer. Fig. 2(B) shows bands assigned to PAni-ES at 291, 415 and $800 \mathrm{~nm}$, which correspond, respectively, to $\pi-\pi^{*}$ transition, the antiligand $\left(\pi^{*}\right)$ transition in the polaronic band, and the polaronic band state due to $\mathrm{HCl}$ doping $[23,31]$. Interactions between PAni-ES and AuNPs can be inferred by the overlapping band at 580 and $800 \mathrm{~nm}$, by comparing with the spectra of the individual components in Fig. S1 and S2 in the Supplementary Material. Further interaction among components is observed in the LbL film with a trilayer architecture in Fig. 2(D), as PAni-ES, AuNPs and $\mathrm{Na}^{+}-\mathrm{MMT}$ are combined. The spectra contain PAni-ES bands at 410 and $780 \mathrm{~nm}$, which are shifted in relation to the same bands in PAni-ES/AuNPs LbL films (at 415 and $800 \mathrm{~nm})$.

In order to understand the effects of incorporation of AuNPs under LbL films of PAni-ES and $\mathrm{Na}^{+}-\mathrm{MMT}$ LbL films, it has been characterized the films using three other methods, namely, X-ray diffraction (XRD), Fourier transform infrared (FTIR) spectroscopy, and Raman spectroscopy. According to the literature [23], the patterns for LbL films of PAni-ES display a large peak at $2 \theta=13.2^{\circ}$, typical of an amorphous polymeric structure. When AuNPs are present, however, no such a peak appears, as indicated in Fig. 3. We hypothesize that incorporation of AuNPs causes uncoiling of PAniES chains. Incorporation of AuNPs also affected the packing of $\mathrm{Na}^{+}-$ MMT LbL films. The peaks at $2 \theta=6.8^{\circ}$ and $2 \theta=7.3$ for PEI $/ \mathrm{Na}^{+}-\mathrm{MMT}$ and PAni-ES/Na+ ${ }^{+}$-MMT LbL films [23] were shifted to $2 \theta=6.4$ and $6.2^{\circ}$ for AuNPs/Na ${ }^{+}-\mathrm{MMT}$ and PAni-ES/AuNPs $/ \mathrm{Na}^{+}-\mathrm{MMT}$, respectively. The interlayer spacing calculated using Bragg equation is $1.37 \mathrm{~nm}$ for the AuNPs/Na+-MMT LbL film and $1.41 \mathrm{~nm}$ for the PAniES/AuNPs $/ \mathrm{Na}^{+}-\mathrm{MMT}$ LbL film. This increased interlayer spacing in comparison with the LbL films without AuNPs (see Table 1) is indicative of intercalation of PAni-ES on the $\mathrm{Na}^{+}-\mathrm{MMT}$ galleries. Another indication of this intercalation is the decrease and almost disappearance of the peak due to $\mathrm{Na}^{+}$-MMT. The molecular level interaction of AuNPs with PAni-ES, which provides the strong effect on the clay structure, is also confirmed by FTIR and Raman spectra.

Fig. 4 shows the transmission FTIR spectra for LbL films with 10 bilayers of PAni-ES/AuNPs and AuNPs/Na+-MMT, and 10 trilayers of PAni-ES/AuNPs/Na ${ }^{+}-\mathrm{MMT}$. A comparison of the band changes and their assignment in the presence and absence of AuNPs are presented in Tables 2 and 3. The main changes in the spectrum of AuNPs/Na+-MMT LbL film are associated with the absence of typical bands for $\mathrm{Na}^{+}-\mathrm{MMT}$, for which only one intense band at $1039 \mathrm{~cm}^{-1}$ was assigned. The main change in the spectra of PAniES/AuNPs and PAni-ES/AuNPs/Na+-MMT is the shift in the peaks at
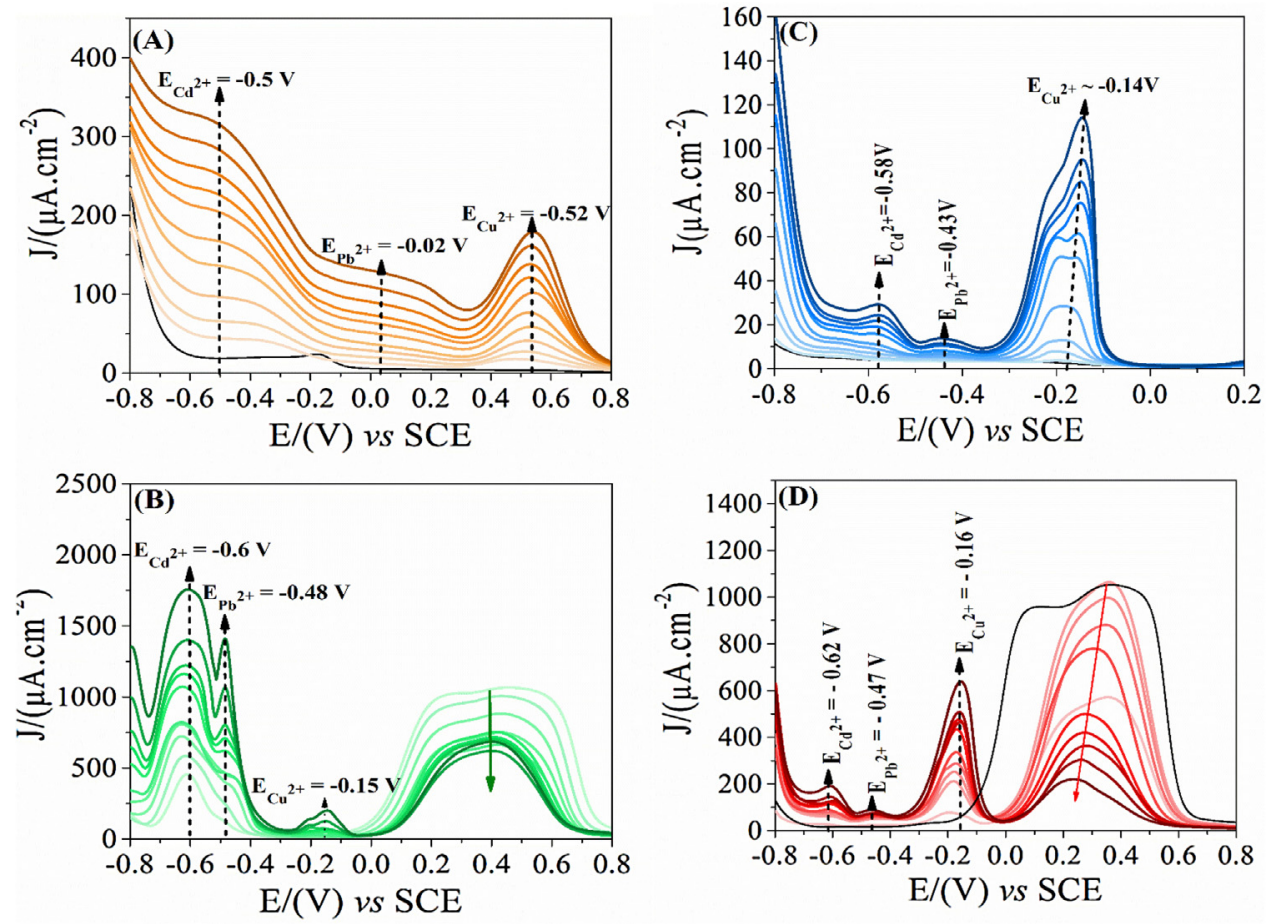

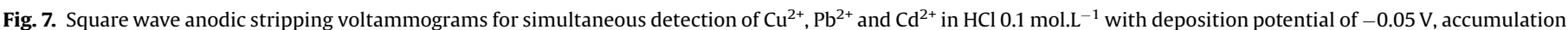

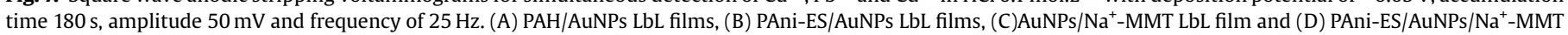
nanocomposite film. All the films have 30 -layers and were deposited on ITO plates. 
1170 and $1314 \mathrm{~cm}^{-1}$ assigned to $\mathrm{N}=\mathrm{Q}=\mathrm{N}$ and $\mathrm{N}-\mathrm{B}-\mathrm{N}$ (Q corresponding to quinoid rings and $\mathrm{B}$ to benzenoid rings) [32-35]. The interaction between PAni-ES and AuNPs in the LbL films of PAni-ES/ AuNPs and PAni-ES/AuNPs/Na ${ }^{+}-\mathrm{MMT}$ is inferred by the peak shift at $1246 \mathrm{~cm}^{-1}$ to $1230 \mathrm{~cm}^{-1}$ with incorporation of AuNPs. More specifically, this peak corresponds to the radical cation stretching $\mathrm{C}-\mathrm{N}^{+}\left(\mathrm{Q}=\mathrm{N}^{+} \mathrm{H}-\mathrm{B}\right.$ and $\left.\mathrm{B}-\mathrm{N}^{+} \mathrm{H}-\mathrm{B}\right)$, suggesting dipole interaction between PAni-ES and sulfonic groups $\left(\mathrm{SO}_{3}{ }^{-}\right)$of thiol ligand from AuNPs [28].

Consistent with FTIR results, the Raman spectra in Fig. 5(A) and 5(B) show that AuNPs affected PAni-ES properties. Bands of PAni-ES are shifted owing to a conformational change in the polymer chain. We have observed such effect in a previous work comparing the peak at $1335 \mathrm{~cm}^{-1}$ for the polaronic state of PAni-ES/PVS and PAni-ES/Na+-MMT LbL films [23], which was small shifted to $1337 \mathrm{~cm}^{-1}$, not affecting the conductivity PAniES properties. On the other hand, a significant change was observed in the bands at 1473 and $1476 \mathrm{~cm}^{-1}$ for PAni-ES/AuNPs and PAni-ES/AuNPs/Na ${ }^{+}-M M T$, respectively, which appear at $1488 \mathrm{~cm}^{-1}$ for PAni-ES/PVS and PAni-ES/Na ${ }^{+}-\mathrm{MMT}$ LbL films [23]. We suggest that this change is associated with an extended polymer chain. The characteristic Raman bands of $\mathrm{Na}^{+}$-MMT cannot be observed, and a decrease in the background intensity due to the incorporation of AuNPs, when compared to LbL film without AuNPs (results not shown). The optical images reveal large aggregates (in the micrometer scale) in some regions, similarly to the AFM images discussed later. Therefore, taking advantage of Raman spectrograph coupled to optical microscopy, we mark in Fig. 5 the spots from where the Raman spectra were taken, i.e., from smooth regions or from aggregates. As expected, the aggregates present a slightly stronger signal than the smooth regions, but this irregularity does not affect the PAni-ES properties, since there were no significant shift in the polaronic band of PAni-ES. In this case, the effect of AuNPs on PAni-ES properties is only direct linked with some shifted bands of PAni-ES that promote a conformational change in polymer chain.

Consistent with optical Raman images, the AFM analysis reveal large aggregates (in the micrometer scale) in some regions, this irregularity caused an increase in roughness of LbL fims. Observed from the AFM images Fig. 6(A)-(D) that the LbL films show a good distribution along the surface, but with largest irregular regions, consequently, increasing the roughness of LbL films. The rootmean square (RMS) roughness was 21.7 and $70.6 \mathrm{~nm}$ for AuNPs/ $\mathrm{Na}^{+}-\mathrm{MMT}$ and PAni-ES/AuNPs while the roughness of the LbL films of PAni-ES/AuNPs/ $\mathrm{Na}^{+}-\mathrm{MMT}$ is $78.0 \mathrm{~nm}$. The increase in roughness for different combinations of LbL films AuNPs/Na ${ }^{+}-$ MMT, PAni-ES/AuNPs and PAni-ES/AuNPs/Na ${ }^{+}-\mathrm{MMT}$ films results in an increased electrochemical intensity (electrochemical analysis by square wave anodic stripping voltammetry to be presented later on), consequently, in a low limit of detection for analysis.

\subsection{Electrochemical Sensor Characterization}

The electrochemical activity of the LB films can be exploited to detect metal ions using SWAS voltammetry shown in Fig. 7(A) to through (D) shows the SWAS voltammograms of PAH/AuNPs, PAni-ES/AuNPs, AuNPs/Na ${ }^{+}-\mathrm{MMT}$, and PAni-ES/ AuNPs $/ \mathrm{Na}^{+}-\mathrm{MMT}$, respectively. All the electrodes were able to discriminate the three metal ions, as indicated by the oxidation peaks. It is possible to observed that the incorporation of AuNPs in the LbL films caused a considerable increase in the current intensity in the voltammograms compared to the literature [23]. Furthermore, a higher resolution for separation of the peaks has been achieved, when compared to the LbL films without AuNPs. The LOD was calculated according to IUPAC, 3s/ $S$, in which $S$ the standard deviation of the response and $S$ is the sensitivity. In our work, the analysis for metal ions detection was made by ten measurements for each architecture sensors to obtain the LOD results and verify the reproducibility for the sensors.The current peak, from which the sensitivity and limit of detection (LOD) of the analytical curves in Fig. 8(A)-(C) were determined, increases with the metal ion concentration. These values are given in Table 4, where a comparison is made with LbL films of PAni-ES/PVS, PEI/Na+-MMT and PAni-ES/Na+-MMT without AuNPs, and with LOD values in the literature obtained with differential pulse voltammetry (DPV). One can infer that the LbL film of PAni-ES/AuNPs/Na+-MMT was better than the other architectures, judging by the data shown in Table 4 . In all the cases, the peaks for the different electrodes in Fig. 7(B)-(D)
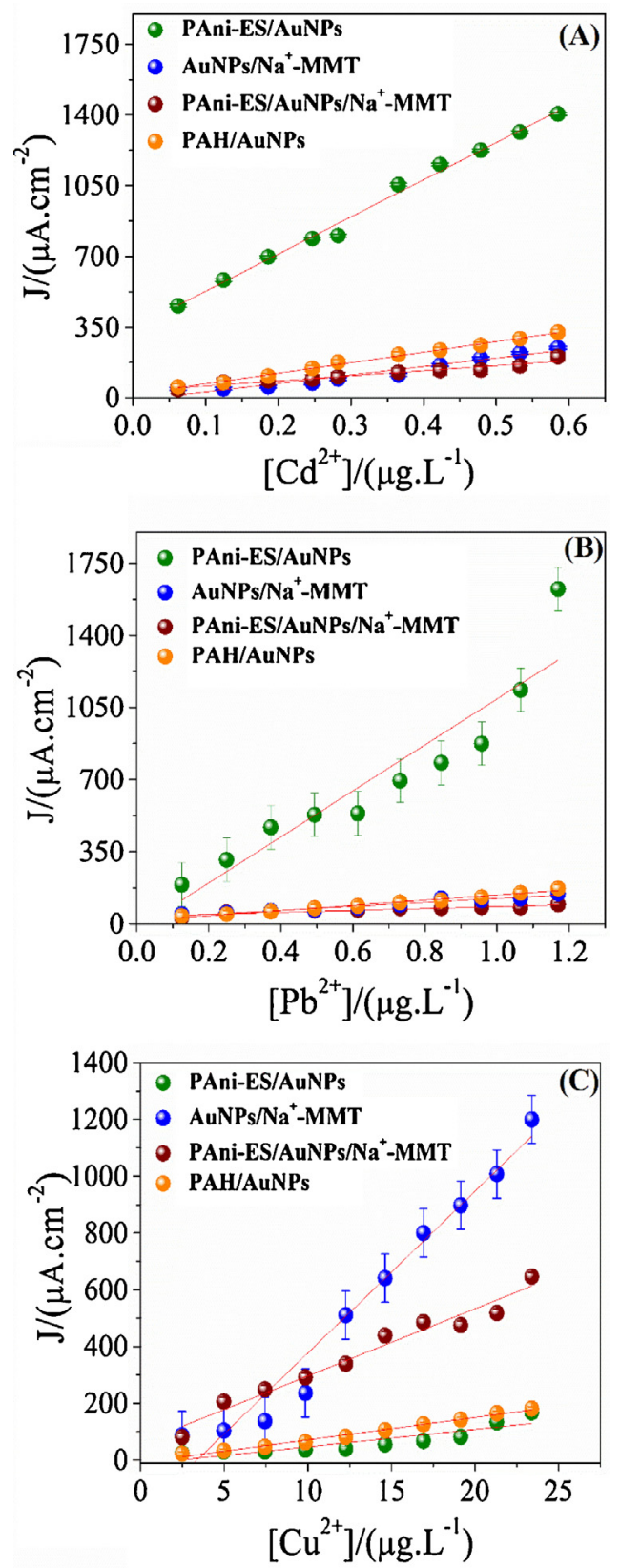

Fig. 8. Analytical curves for detecting metallic ions with electrodes modified with PAni-ES/AuNPs, AuNPs/Na ${ }^{+}-\mathrm{MMT}$ and PAni-ES/AuNPs/Na ${ }^{+}-\mathrm{MMT}$. (A) $\mathrm{Cd}^{2+}$, (B) $\mathrm{Pb}^{2}$ + and $(\mathrm{C}) \mathrm{Cu}^{2+}$. 
Table 4

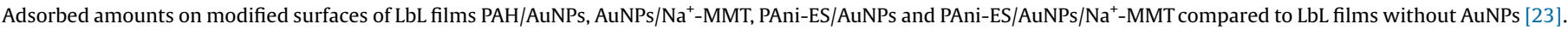

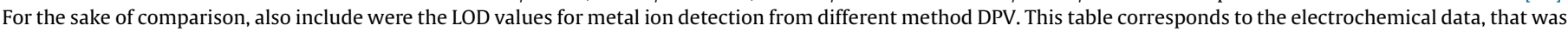
cited by Table 5, now corrected in the text.

\begin{tabular}{|c|c|c|c|c|c|c|}
\hline $\begin{array}{l}\text { Analyte } \\
\text { (metallic ions) }\end{array}$ & Electrode modified & $\begin{array}{l}\text { Sensitivity } \\
\left(\mu \mathrm{Acm}^{-2} \mathrm{mg} \mathrm{L}^{-1}\right)\end{array}$ & $\begin{array}{l}\text { LOD } \\
\left(\mu \mathrm{g} \mathrm{L}^{-1}\right)\end{array}$ & $\begin{array}{l}\text { Electrode modified } \\
\text { (without AuNPs) }\end{array}$ & $\begin{array}{l}\text { LOD } \\
\left(\mu \mathrm{g} \mathrm{L}^{-1}\right)\end{array}$ & Comparison of LOD with the literature $\left(\mu \mathrm{g} \mathrm{L}^{-1}\right)$ \\
\hline $\mathrm{Cd}^{2+}$ & PAH/AuNPs & $18.4 \pm 2.6$ & 0.42 & & & $1.5[41]$ \\
\hline $\mathrm{Pb}^{2+}$ & & $95.5 \pm 1.7$ & 0.06 & - & - & $0.05[41]$ \\
\hline $\mathrm{Cu}^{2+}$ & & $8.0 \pm 2$ & 0.99 & & & - \\
\hline $\mathrm{Cd}^{2+}$ & AuNPs/Na+-MMT & $420 \pm 2$ & 0.02 & & 310 & $0.10[42]$ \\
\hline $\mathrm{Pb}^{2+}$ & & $477 \pm 2$ & 0.01 & $\mathrm{PEI} / \mathrm{Na}^{+}-\mathrm{MMT}$ & 1350 & $0.45[42]$ \\
\hline $\mathrm{Cu}^{2+}$ & & $6.1 \pm 0.2$ & 0.11 & & 2090 & $0.20[42]$ \\
\hline $\mathrm{Cd}^{2+}$ & PAni-ES/AuNPs & $251 \pm 1$ & 0.02 & & 170 & $0.012[40]$ \\
\hline $\mathrm{Pb}^{2+}$ & & $120 \pm 68$ & 0.17 & PAni-ES/PVS & 910 & $0.029[40]$ \\
\hline $\mathrm{Cu}^{2+}$ & & $57,02 \pm 0.76$ & 0.04 & & 750 & - \\
\hline $\mathrm{Cd}^{2+}$ & PAni-ES/AuNPs/Na ${ }^{+}-\mathrm{MMT}$ & $521 \pm 1$ & 0.01 & & 0.27 & $0.01[43]$ \\
\hline $\mathrm{Pb}^{2+}$ & & $125 \pm 1$ & 0.009 & PAni-ES/Na+-MMT & 0.28 & $0.02[43]$ \\
\hline $\mathrm{Cu}^{2+}$ & & $23.7 \pm 0.2$ & 0.02 & & 0.09 & $0.02[43]$ \\
\hline
\end{tabular}

appear approximately at $\mathrm{E}=-0.6 \mathrm{~V}, \mathrm{E}=-0.4 \mathrm{~V}$ and $\mathrm{E}=-0.1 \mathrm{~V}$ for $\mathrm{Cd}^{2+}, \mathrm{Pb}^{2+}$ and $\mathrm{Cu}^{2+}$, respectively. When compared the values of peak potentials, there is a considerable shift from what is expected from the literature. For the LbL films without AuNPs, which should be approximately $\mathrm{E}=-0.7 \mathrm{~V}, \mathrm{E}=-0.1 \mathrm{~V}$ and $\mathrm{E}=+0.3 \mathrm{~V}$ for the same species $[22,23,36-40]$. In addition, observed in Fig. 7(B) and (D) one peak in approximately $\mathrm{E}=0.4 \mathrm{~V}$ that corresponds to emeraldine salt conducting form. Observed that the peak characteristic of PAni-ES decrease with the increase of metal ions concentration, we suggest that this effect occurs due to oxidation of AuNPs immobilized on electrode surface. In summary, was possible to detection of the three metal ions simultaneously and a clearer distinction was achieved for separation peaks without the overlap with the PAni-ES peaks, due to the presence of AuNPs. Compared to differents architectures for the sensors depicted in this work, we conclude that the electrode coated with PAni-ES/AuNPs/Na ${ }^{+}-$ MMT is the most efficient in detecting the three metal ions, because the separation is more efficient and the LOD is lower than for the LbL films made of PAni-ES and $\mathrm{Na}^{+}-\mathrm{MMT}$ without of AuNPs. This superior performance of LbL films composed of PAni-ES/AuNPs/ $\mathrm{Na}^{+}-\mathrm{MMT}$ points to synergy between the materials for this sensor and high electrocatalytic activity promoted by AuNPs.

\subsection{Conclusions}

The presence of AuNPs in the LbL films of PAni-ES and $\mathrm{Na}^{+}-$ MMT promoted higher stability in comparison to LbL films without AuNPs. We also suggest PAni-ES and AuNPs caused the extension of the polymer chain favoring the ion intercalation into the clay interlayer spaces as supported by X-ray diffraction data that revealed an increased basal spacing of clay layers. FTIR and Raman spectra confirmed the effect of AuNPs on PAni-ES properties, with a significant shift of its polaronic band. Evidenced by the AFM results, the increase of the root-mean squared roughness with the incorporation of AuNPs in LbL films, containing of PAni-ES and $\mathrm{Na}^{+}-\mathrm{MMT}$, enhanced the electrocatalytic effect for detecting $\mathrm{Cu}^{2+}, \mathrm{Pb}^{2+}$ and $\mathrm{Cd}^{2+}$ metal ions simultaneously, yielding a higher performance for the sensors with a lower detection limit, from $\mathrm{mg} \mathrm{L}^{-1}$ to $\mu \mathrm{gL}^{-1}$.

\section{Acknowledgements}

This work was supported by FAPESP (Grants 2011/00733-2 and 2012/16158-0), CNPQ (Grant 473222/2012-4), CAPES and NBioNet network. The authors are also grateful to Prof. Osvaldo N. Oliveirajr for helpful discussions, Sabrina Aléssio Camacho for the help with the Raman measurements and Rafael Parra Ribeiro for the help with XRD analysis.

\section{Appendix A. Supplementary data}

Supplementary data associated with this article can be found, in the online version, at http://dx.doi.org/10.1016/j. electacta.2017.03.135.

\section{References}

[1] G. Decher, Fuzzy Nanoassemblies: Toward Layered Polymeric Multicomposites, Science 277 (1997) 1232-1237.

[2] B. Tieke, Coordinative supramolecular assembly of electrochromic thin films, Curr. Opin. Colloid Interface Sci. 16 (2011) 499-507, doi:http://dx.doi.org/ 10.1016/j.cocis.2011.07.002

[3] K. Ariga, Y. Yamauchi, G. Rydzek, Q. Ji, Y. Yonamine, K.C.-W. Wu, J.P. Hill, Layerby-layer Nanoarchitectonics: Invention, Innovation, and Evolution, Chem. Lett. 43 (2014) 36-68.

[4] K. Ariga, Q. Ji, W. Nakanishi, J.P. Hill, Thin Film Nanoarchitectonics, J. Inorg. Organomet. Polym. Mater. 25 (2015) 466-479.

[5] Q. Xi, X. Chen, D.G. Evans, W. Yang, Gold Nanoparticle-Embedded Porous Graphene Thin Films Fabricated via Layer-by-Layer Self-Assembly and Subsequent Thermal Annealing for Electrochemical Sensing, Langmuir 28 (2012) 9885-9892

[6] H. Hwang, P. Joo, M.S. Kang, G. Ahn, J.T. Han, B.-S. Kim, J.H. Cho, Highly Tunable Charge Transport in Layer-by-Layer Assembled Graphene Transistors, ACS Nano 6 (2012) 2432-2440.

[7] S.K. Saha, A. Guchhait, A.J. Pal, Organic/inorganic hybrid pn-junction between copper phthalocyanine and CdSe quantum dot layers as solar cells, J. Appl. Phys. 112 (2012) 044507.

[8] R.F. de Oliveira, M.L. de Moraes, O.N. Oliveira, M. Ferreira, Exploiting Cascade Reactions in Bienzyme Layer-by-Layer Films, J. Phys. Chem. C 115 (2011) 19136-19140.

[9] R.J. El-khouri, R. Szamocki, Y. Sergeeva, O. Felix, G. Decher, Multifunctional Layer-by-Layer Architectures for Biological Applications, Funct. Polym. Films, Wiley-VCH, 2011, pp. 11-72.

[10] B. Koo, H. Baek, J. Cho, Control over Memory Performance of Layer-by-Layer Assembled Metal Phthalocyanine Multilayers via Molecular-Level Manipulation, Chem. Mater. 24 (2012) 1091-1099.

[11] T. Boudou, T. Crouzier, K. Ren, G. Blin, C. Picart, Multiple Functionalities of Polyelectrolyte Multilayer Films: New Biomedical Applications, Adv. Mater. 22 (2010) 441-467.

[12] S.A. Camacho, P.H.B. Aoki, C.J.L. Constantino, A.M. Pires, Sprayed films of europium complexes toward light conversion devices, J. Lumin. 153 (2014) $272-280$.

[13] R. Sardar, A.M. Funston, P. Mulvaney, R.W. Murray, Gold Nanoparticles: Past, Present, and Future, Langmuir 25 (2009) 13840-13851.

[14] P. Ghosh, G. Han, M. De, C. Kim, V. Rotello, Gold nanoparticles in delivery applications, Adv. Drug Deliv. Rev. 60 (2008) 1307-1315.

[15] H. AlQahtani, M. Sugden, D. Puzzovio, L. Hague, N. Mullin, T. Richardson, M. Grell, Highly sensitive alkane odour sensors based on functionalised gold nanoparticles, Sens. Actuators B Chem. 160 (2011) 399-404.

[16] J.-W. Park, J.S. Shumaker-Parry, Structural Study of Citrate Layers on Gold Nanoparticles: Role of Intermolecular Interactions in Stabilizing Nanoparticles, J. Am. Chem. Soc. 136 (2014) 1907-1921.

[17] C. Medina-Plaza, L.N. Furini, C.J.L. Constantino, J.A. de Saja, M.L. RodriguezMendez, Synergistic electrocatalytic effect of nanostructured mixed films 
formed by functionalised gold nanoparticles and bisphthalocyanines, Anal Chim. Acta 851 (2014) 95-102.

[18] T. Berzina, A. Pucci, G. Ruggeri, V. Erokhin, M.P. Fontana, Gold nanoparticlespolyaniline composite material: Synthesis, structure and electrical properties, Synth. Met. 161 (2011) 1408-1413.

[19] A. Safavi, E. Farjami, Construction of a carbon nanocomposite electrode based on amino acids functionalized gold nanoparticles for trace electrochemical detection of mercury, Anal. Chim. Acta 688 (2011) 43-48.

[20] H. Rao, M. Chen, H. Ge, Z. Lu, X. Liu, P. Zou, X. Wang, H. He, X. Zeng, Y. Wang, A novel electrochemical sensor based on Au@PANI composites film modified glassy carbon electrode binding molecular imprinting technique for the determination of melamine, Biosens. Bioelectron. 87 (2017) 1029-1035.

[21] H.L. Nguyen, H.H. Cao, D.T. Nguyen, V.-A. Nguyen, Sodium Dodecyl Sulfate Doped Polyaniline for Enhancing the Electrochemical Sensitivity of Mercury Ions, Electroanalysis (2016).

[22] A. De Barros, C.J.L. Constantino, J.R.R. Bortoleto, N.C. Da Cruz, M. Ferreira, Incorporation of gold nanoparticles into Langmuir-Blodgett films of polyaniline and montmorillonite for enhanced detection of metallic ions, Sens. Actuators B Chem. 236 (2016) 408-417.

[23] A. de Barros, M. Ferreira, C.J.L. Constantino, M. Ferreira, Nanocomposites based on LbL films of polyaniline and sodium montmorillonite clay, Synth. Met. 197 (2014) 119-125.

[24] Y. Umemura, Y. Onodera, A. Yamagishi, Layered structure of hybrid films of an alkylammonium cation and a clay mineral as prepared by the LangmuirBlodgett method, Thin Solid Films 426 (2003) 216-220.

[25] J.H. Cheung, W.B. Stockton, M.F. Rubner, Molecular-level processing of conjugated polymers. 3. Layer-by-layer manipulation of polyaniline via electrostatic interactions, Macromolecules 30 (1997) 2712-2716.

[26] I. Gofberg, D. Mandler, Preparation and comparison between different thiolprotected Au nanoparticles, J. Nanoparticle Res. 12 (2009) 1807-1811.

[27] S.W. Karickhoff, G.W. Bailey, Optical absorption spectra of clay minerals, Clays Clay Min. 21 (1973) 59-70.

[28] X. Zou, H. Bao, H. Guo, L. Zhang, L. Qi, J. Jiang, L. Niu, S. Dong, Mercaptoethane sulfonate protected, water-soluble gold and silver nanoparticles: Syntheses, characterization and their building multilayer films with polyaniline via iondipole interactions, J. Colloid Interface Sci. 295 (2006) 401-408.

[29] C.-W. Chiu, J.-J. Lin, Self-assembly behavior of polymer-assisted clays, Prog. Polym. Sci. 37 (2012) 406-444.

[30] N. Bitinis, M. Hernandez, R. Verdejo, J.M. Kenny, M.A. Lopez-Manchado, Recent Advances in Clay/Polymer Nanocomposites, Adv. Mater. 23 (2011) 5229-5236.

[31] G. Ćirić-Marjanović, Recent advances in polyaniline research: Polymerization mechanisms, structural aspects, properties and applications, Synth. Met. 177 (2013) 1-47.
[32] G.M. do Nascimento, V.R.L. Constantino, R. Landers, M.L.A. Temperini, Aniline Polymerization into Montmorillonite Clay: A Spectroscopic Investigation of the Intercalated Conducting Polymer, Macromolecules 37 (2004) 9373-9385.

[33] P. Thomas, K. Dwarakanath, K.B.R. Varma, In situ synthesis and characterization of polyaniline-CaCu3Ti4012 nanocrystal composites, Synth. Met. 159 (2009) 2128-2134.

[34] B.N. Narayanan, R. Koodathil, T. Gangadharan, Z. Yaakob, F.K. Saidu, S. Chandralayam, Preparation and characterization of exfoliated polyaniline montmorillonite nanocomposites, Mater. Sci. Eng. B 168 (2010) 242-244.

[35] A.F. Baldissera, J.F. Souza, C.A. Ferreira, Synthesis of polyaniline/clay conducting nanocomposites, Synth. Met. 183 (2013) 69-72.

[36] A. de Barros, M. Ferreira, C.J. Leopoldo Constantino, J.R. Ribeiro Bortoleto, M. Ferreira, Synergy between Polyaniline and OMt Clay Mineral in LangmuirBlodgett Films for the Simultaneous Detection of Traces of Metal Ions, Acs Appl. Mater. Interfaces 7 (2015) 6828-6834.

[37] M.B. Gumpu, S. Sethuraman, U.M. Krishnan, J.B.B. Rayappan, A review on detection of heavy metal ions in water -An electrochemical approach, Sens. Actuators B Chem. 213 (2015) 515-533.

[38] Y. Dong, Y. Ding, Y. Zhou, J. Chen, C. Wang, Differential pulse anodic stripping voltammetric determination of $\mathrm{Pb}$ ion at a montmorillonites/polyaniline nanocomposite modified glassy carbon electrode, J. Electroanal. Chem. 717 (2014) 206-212.

[39] L. Zhu, L. Xu, B. Huang, N. Jia, L. Tan, S. Yao, Simultaneous determination of Cd (II) and $\mathrm{Pb}(\mathrm{II})$ using square wave anodic stripping voltammetry at a gold nanoparticle-graphene-cysteine composite modified bismuth film electrode, Electrochimica Acta 115 (2014) 471-477.

[40] R. María-Hormigos, M.J. Gismera, J.R. Procopio, M.T. Sevilla, Disposable screenprinted electrode modified with bismuth-PSS composites as high sensitive sensor for cadmium and lead determination, J. Electroanal. Chem. 767 (2016) $114-122$.

[41] L. Xiao, H. Xu, S. Zhou, T. Song, H. Wang, S. Li, W. Gan, Q. Yuan, Simultaneous detection of $\mathrm{Cd}(\mathrm{II})$ and $\mathrm{Pb}(\mathrm{II})$ by differential pulse anodic stripping voltammetry at a nitrogen-doped microporous carbon/Nafion/bismuth-film electrode, Electrochimica Acta 143 (2014) 143-151.

[42] H. Lin, M. Li, D. Mihailovič, Simultaneous Determination of Copper, Lead, and Cadmium Ions at a Mo6S9-xIx Nanowires Modified Glassy Carbon Electrode Using Differential Pulse Anodic Stripping Voltammetry, Electrochimica Acta 154 (2015) 184-189.

[43] Z. Guo, D. Li, X. Luo, Y. Li, O.-N. Zhao, M. Li, Y. Zhao, T. Sun, C. Ma, Simultaneous determination of trace $\mathrm{Cd}(\mathrm{II}), \mathrm{Pb}(\mathrm{II})$ and $\mathrm{Cu}(\mathrm{II})$ by differential pulse anodic stripping voltammetry using a reduced graphene oxide-chitosan/poly-l-lysine nanocomposite modified glassy carbon electrode, J. Colloid Interface Sci. 490 (2017) 11-22. 